\title{
Value of 99mTc-ECD Single-Photon Emission CT in Diagnosis and Recurrence Prediction of Post-Stroke Epilepsy
}

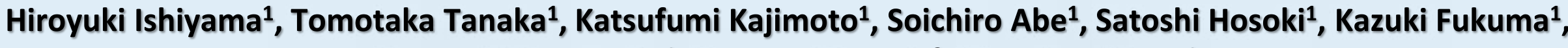 \\ Kazuyuki Nagatsuka1, Kazunori Toyoda², Masafumi Ihara ${ }^{1}$ \\ ${ }^{1}$ Divisions of Neurology and ${ }^{2}$ Cerebrovascular Medicine, Department of Stroke and Cerebrovascular Diseases, \\ National Cerebral and Cardiovascular Center, Suita, Japan
}

\section{INTRODUCTION}

Several studies have shown that ictal Single-Photon Emission CT (SPECT) with 99mTechnetium-Ethyl-Cysteinate-Dimer ( $99 \mathrm{mTC}-\mathrm{ECD}$ ) is a useful method for identifying the epileptogenic zone. However, the association between the hyperperfusion area on SPECT and seizure recurrence remains unclear in the patient with post-stroke epilepsy (PSE).

\section{METHODS}

Study design: A single hospital based, prospective, observational study

Subjects

55 patients with PSE from November 2014 to June 2016.

14 cases without $99 \mathrm{mTC}-\mathrm{ECD}$ SPECT excluded

1 patients with $99 \mathrm{mTC}-\mathrm{ECD}$ SPECT.

Evaluation of Lateralization

Stroke lesion: infarction or hemorrhage on MRI/ CT.

Hyperperfusion area on ictal/(or early postictal) SPECT: visual inspection by two examiners or subtraction ictal SPECT coregistered to MRI (SISCOM) in SPECT.

$>$ epileptiform discharge: spike or sharp wave on EEG within 5 days of seizure.

Analysis

Lateralization concordance of hyperperfusion area and stroke lesion on CT/MRI or epileptiform discharge on EEG.

$>$ Association between the presence of hyperperfusion area and one-year recurrence of seizure by Pearson's chi-square test.

\section{RESULTS}

We included 41 PSE patients. Hyperperfusion was observed in 22 (54\%) patients, of whom 3 (14\%) were detected only by SISCOM.

\begin{tabular}{|c|ccc|} 
& Table 1. Demographics & \\
\hline & Age (mean) & Male (\%) & Hyperperfusion (\%) \\
\hline Total ( $=41)$ & 72 & 61 & 54 \\
\hline Ischemic Stroke $(\mathrm{N}=20)$ & 78 & 70 & 55 \\
Hemorrhage $(\mathrm{N}=19)$ & 70 & 58 & 58 \\
\hline Both $(\mathrm{N}=2)$ & 78 & 0 & 0 \\
\hline
\end{tabular}

The hyperperfusion area was ipsilateral to stroke lesion in 18 (82\%) of the 22 patients, indicating that the lateralization was significantly concordant $(P<0.01)$. All $6(27 \%)$ patients with the epileptiform discharge on EEG had ipsilateral hyperperfusion area $(P=0.01)$.

Table 2. Lateralization Concordance Lateralization

\begin{tabular}{|c|c|c|c|c|c|c|c|}
\hline & \multicolumn{7}{|c|}{ Lateralization } \\
\hline & \multicolumn{2}{|c|}{ Hyperperfusion } & \multicolumn{2}{|c|}{ Stroke lesion } & \multicolumn{3}{|c|}{ epileptiform discharge } \\
\hline & Rt & $\mathrm{Lt}$ & Rt & Lt & Rt & $\mathrm{Lt}$ & Negative \\
\hline \multirow[t]{3}{*}{ Total $(\mathrm{N}=22)$} & 12 & 10 & 10 & 12 & 4 & 2 & 16 \\
\hline & \multicolumn{7}{|c|}{$\begin{array}{c}\text { Lateralization concordance ratio } \\
\text { of ECD-SPECT, } n(\%)\end{array}$} \\
\hline & \multicolumn{3}{|c|}{ Stroke lesion } & \multicolumn{4}{|c|}{ epileptiform discharge } \\
\hline Total $(\mathrm{N}=22)$ & \multicolumn{3}{|c|}{$18 / 22(82)$} & \multicolumn{4}{|c|}{$6 / 6(100)$} \\
\hline
\end{tabular}

Recurrence of epilepsy in one year was identified in 12 (29\%) patients. The median seizure interval was 195 day (IQR 166.5-307). Antiepileptiform drugs were taken by 10 (83\%) patients. The recurrence ratio was not associated with presence of hyperperfusion on $99 \mathrm{mTC}-E C D$ SPECT $(P=0.32)$.

Figure. Recurrence of Epilepsy

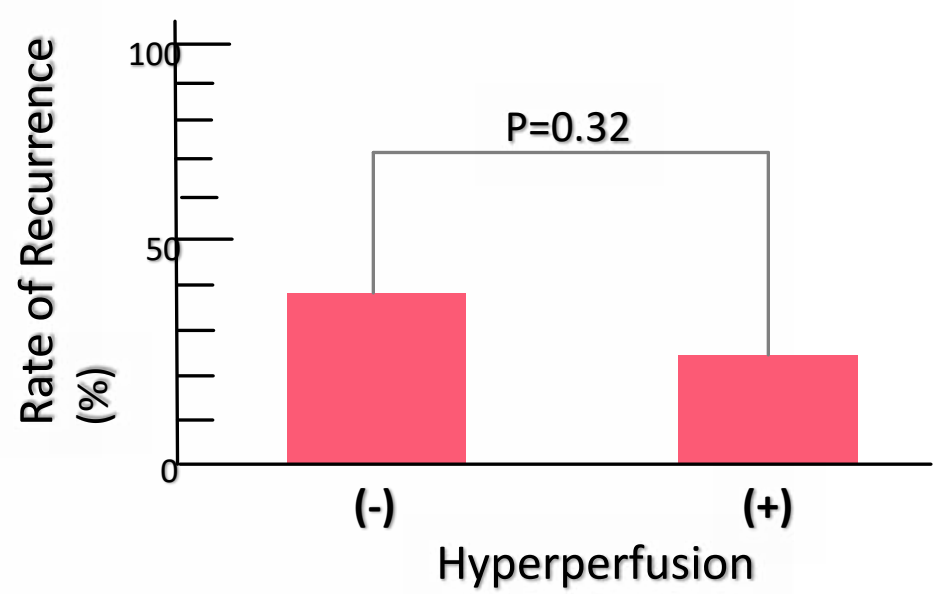

\section{DISCUSSION}

$>$ In this research, we found that in the patients of PSE, hyperperfusion was ipsilateral to stroke lesion on MRI/CT or to epileptiform discharge on EEG, which was considered to be focus of epilepsy. It is often difficult to find hyperperfusion in PSE patients since hypoperfusion area associated with stroke lesion is contiguous. Therefore, SISCOM may be useful for the identification of hyperperfusion area in PSE patients.

$>$ We also assessed whether the presence of hyperperfusion was associated with recurrence of epilepsy as the increased perfusion may reflect increased epileptic activity. However, there was no association between hyperperfusion and recurrence of epilepsy. We need further investigation such as distribution or intensity of hyperperfusion.

\section{CONCLUSIONS}

99mTc-ECD SPECT is as useful as other diagnostic modalities for identifying epileptic activity in PSE although it does not predict one-year recurrence of PSE.

\section{REFFERENCES}

Ruben I. Kuzniecky. The Journal of the American Society for Experimental NeuroTherapeutics 2 (2005) 384-393.

C. la Fougère et al. Epilepsy \& Behavior 15 (2009) 50-55. 